\title{
AVALIAÇÃO DA EXTENSÃO DA NEOPLASIA EM CÂNCER DA PRÓSTATA: VALOR DO PSA, DA PERCENTAGEM DE FRAGMENTOS POSITIVOS E DA ESCALA DE GLEASON
}

\author{
Antonio Carlos Calvete, Miguel Srougi, luciano João Nesrallah*, \\ Marcos Francisco Dali'Oglio, Valdemar Ortiz \\ Trabalho realizado na Disciplina de Urologia - Universidade Federal de São Paulo - \\ Escola Paulista de Medicina, São Paulo, SP.
}

RESUMO - OBJETIVos. Avaliar o comportamento da percentagem de fragmentos da biópsia prostática, guiada por ultra-sonografia transretal, na previsão de doença extraprostática em pacientes com adenocarcinoma localizado de próstata e, também, comparar a eficiência deste parâmetro com aqueles obtidos pela avaliação do PSA sérico e escala de Gleason pré-operatórios.

Métodos. Foi realizado estudo retrospectivo não controlado de $\mathbf{5 2 2}$ pacientes portadores de adenocarcinoma localizado de próstata e submetidos a tratamento cirúrgico através da prostatectomia radical retropúbica. A idade dos pacientes variou entre 42 a 76 anos, média de 62,44 anos. Todos os pacientes foram submetidos à ultra-sonografia transretal com biópsia prostática (direta da lesão e/ou área suspeita e sextante) antes da prostatectomia radical retropúbica. Os 522 pacientes foram divididos em grupos quanto à percentagem de fragmentos positivos encontrados na biópsia, que foram correlacionados com os achados anatomopatológicos de doença intraprostática (confinada à glândula) e extraprostática (invasão tumoral da gordura periprostática e/ou invasão de colo vesical e/ou invasão das vesículas seminais e/ou linfonodos pélvicos positivos), do espécime cirúrgico.
Resultados. $\mathrm{Na}$ análise da percentagem de fragmentos positivos os grupos $\mathbf{~ G I ( 0 - 2 5 \% ) , ~ G 2 ( 2 6 - 5 0 \% ) ~ e ~ G 3 ( 5 I - 7 5 \% ) ~ a p r e s e n t a - ~}$ ram incidência de doença intraprostática duas a três vezes maior do que extraprostática. No entanto, quando mais de $75 \%$ dos fragmentos de biópsia foram positivos (G4 76-100\%), a relação se inverteu, ocorrendo uma predominância de doença extrapros. tática sobre a doença intraprostática. Neste grupo $56,98 \%$ dos pacientes evidenciaram doença extraprostática. Houve diferença, estatisticamente, significativa da doença extraprostática entre os grupos G3 e G4 ( $p=0,0068)$.

Conclusónes. Quando comparamos os três parâmetros para avaliar a ordem de eficiência em prever doença extraprostática, verificamos que o PSA pré-operatório foi o mais discriminante $(p=0,000000)$, seguido pela escala de Gleason da biópsia prostática $(p=0,000003)$ e pela percentagem de biópsias positivas $(p=0,000574)$.

Unitermos: Próstata. Antígeno prostático específico. Neoplasia prostática. Biópsia.

\section{INTRODUÇÃO}

Nas últimas quatro décadas, o câncer da próstata tornou-se um problema relevante de saúde pública. Éa doença maligna mais comum do homem nos Estados Unidos e a segunda causa mais comum de morte por câncer ${ }^{1,2}$. Foi estimado, que no período de 1985 a 2000 , haverá um aumento de $37 \%$ de mortes por ano e um aumento de $90 \%$ de novos casos diagnosticados nesse país ${ }^{3}$. No Brasil, segundo dados oficiais fornecidos em 1999 pelo Instituto $\mathrm{Na}$ cional do Câncer - INCA, foram identificados cerca de 18,49 novos casos por 100.000 habitantes, ficando ocâncer de próstata em segundo lugar após o câncer de pulmão, cuja incidênciaé de 18,89 novos casos por 100 mil habitantes.

* Correspondência:

R. Barata Ribeiro, 414 CJ 25 - 01308-000 São Paulo - SP - nesrallahuro@uol.com.br
$\mathrm{Na}$ apresentação inicial dos pacientes com o diagnóstico de câncer de próstata, $58 \%$ têm câncer localizado, 15\% têm envolvimento regional e $16 \%$ têm metástases à distância, a maioria assintomática ou com sintomas de obstrução urinária baixa ${ }^{4}$.

O PSA é considerado o mais importante marcador para detectar, estagiaremonitorizaro câncer de próstata ${ }^{5}$. Estudos demonstraram que oPSA pré-operatório pode predizer a existência de doença extraprostática ${ }^{6,7,8}$. $O$ sistema de Gleason tem sido introduzido também para ajudar a prever a evolução e predizer o estágio patológico. Sabemos que lesões com escore de Gleason 2-4 (bem diferenciados) têm um comportamento mais indolente e as de escore 7-10 (indiferenciados) são agressivos, respondendo mal às diferentes opções de tratamento.

A biópsia sextante da próstata guiada por ultra-sonografia transretal é o método mais recomendado, na prática, para diagnosticar câncer de próstata. Vários autores têm tentado quantificar, de diversas maneiras, os dados da biópsia prostática, na tentativa de identificar achados pré-operatórios que possam ajudar a diminuir o subestagiamento e prever extensões extracapsulares ou falha biológica. Estas biópsias, quando adequadamente realizadas, são fonte de inúmeras informações, pois podem ser usadas para 0 estudo da cápsula, tecidos periprostáticos, espaços perineurais, quantificação de tumor nos fragmentos, podendo estimar, com isto, a multifocalidade, o volume do tumor e a extensão extraprostática ${ }^{9-16}$.

Observamos que existem controvérsias na literatura sobre o real valor da biópsia sistemática sextante na previsão de doença intra ou extraprostática, porém, constatamos um potencial clínico para a utilização da 
biópsia prostática na tentativa de estabelecer o diagnóstico preciso, o prognóstico e até mesmo a conduta terapêutica no câncer de próstata.

Como não existe, até o momento, um método perfeito que permite prever doença órgão confinada, resolvemos estudar, dentre os diversos achados da biópsia prostática, o valor da percentagem de fragmentos positivos. O nosso objetivo ao utilizar esta variável, fácil de obter e relativamente confiável, foi introduzir em clínica um parâmetro adicional que permita predizer, antes da cirurgia, a existência de doença extraprostática. Esta informação poderia, em alguns casos, mudar a estratégia de tratamento de pacientes com câncer localizado da próstata.

\section{Métodos}

Desenho: Estudo retrospectivo, não controlado, de revisão de prontuários clínicos.

Participantes: Foram estudados 522 pacientes com diagnóstico de câncer localizado da próstata e submetidos à prostatectomia radical retropúbica, pelo mesmo cirurgião (M. S.), conforme técnica publicada anteriormente ${ }^{17}$, entre 1986 e 1999. A idade destes pacientes variou entre $42 \mathrm{a}$ 76 anos (média de 62 anos). 0 diagnóstico foi confirmado através da biópsia em sextante realizada sob orientação da ultrasonografia transretal da próstata. Os pacientes foram estagiados conforme a classificação internacional TNM ${ }^{18}$, sendo que 199 pacientes $(38,2 \%)$ portavam doença em estágio TI, $222(42,5 \%)$ apresentavam estágio T2 a-be I0I (19,3\%) em estagio clínico T2cT3a. Em relação aos valores iniciais de PSA, 45 pacientes $(8,6 \%)$ apresentavam valores de antígeno prostático específico entre 0 $4,0 \mathrm{ng} / \mathrm{ml}, 244$ pacientes (46,7\%), valores entre $4, I-10,0 \mathrm{ng} / \mathrm{ml},|6|$ pacientes $(30,8 \%)$ tinham entre $10,1-20,0 \mathrm{ng} / \mathrm{ml} \mathrm{e} 72$ pacientes $(13,7 \%)$ portavam valores iniciais de PSA $>20 \mathrm{ng} / \mathrm{ml}$. Os valores extremos de PSA inicial foram $0,3 \mathrm{ng} / \mathrm{ml}$ como limite inferior e $72,0 \mathrm{ng} / \mathrm{ml}$ como limite superior, a média de $12,5 \mathrm{ng} / \mathrm{ml}$. Segundo o escore de Gleason da biópsia, I 39 pacientes $(26,6 \%)$ apresentavam escore entre 2-4, 351 $(67,2 \%)$ entre $5-7$ e $32(6,1 \%)$ portavam o escore de Gleason entre 8-10.

\begin{tabular}{|c|c|c|c|c|c|c|}
\hline \multirow[t]{2}{*}{ Grupos } & \multirow{2}{*}{$\begin{array}{c}\text { PSA } \\
(\mathrm{ng} / \mathrm{ml})\end{array}$} & \multicolumn{2}{|c|}{ DIP } & \multicolumn{2}{|c|}{ DEP } & \multirow[t]{2}{*}{$n$} \\
\hline & & $\mathrm{n}$ & $\%$ & $\mathrm{n}$ & $\%$ & \\
\hline $\mathrm{Gl}$ & $0-4,0$ & 39 & 86,67 & 6 & 13,33 & 45 \\
\hline G2 & $4, I-10,0$ & 179 & 73,36 & 65 & 26,64 & 244 \\
\hline G3 & $|0|-20,0$, & 94 & 58,39 & 67 & 41,61 & $16 \mid$ \\
\hline G4 & $>20,0$ & 34 & 47,22 & 38 & 52,78 & 72 \\
\hline Total & 346 & 66,28 & 176 & 33,72 & 522 & \\
\hline
\end{tabular}

PSA-antígeno prostático específico; DIP-doença intraprostática; DEP-doença extraprostática; n-número de pacientes Pearsonqui-quadrado $\quad \mathrm{p}=0,00000$

Tabela 2- Correlação entre a escala de Gleason da biópsia e o câncer intra e extraprostático

\begin{tabular}{|c|c|c|c|c|c|c|}
\hline \multirow[t]{2}{*}{ Grupos } & \multirow{2}{*}{$\begin{array}{l}\text { Escala de } \\
\text { Gleason }\end{array}$} & \multicolumn{2}{|c|}{ DIP } & \multicolumn{2}{|c|}{ DEP } & \multirow[t]{2}{*}{$\mathrm{n}$} \\
\hline & & $n$ & $\%$ & $n$ & $\%$ & \\
\hline GI & $2-4$ & 109 & 78,42 & 30 & 21,58 & 139 \\
\hline G2 & $5-6$ & 190 & 67,86 & 90 & 34,14 & 280 \\
\hline G3 & $7-10$ & 47 & 45,63 & 56 & 54,37 & 103 \\
\hline Total & 346 & 66,28 & 176 & 33,72 & 522 & \\
\hline
\end{tabular}

DIP-doençaintraprostática; DEP-doença extraprostática; $n$-números de pacientes

$$
\text { Pearsonqui-quadrado } \quad \mathrm{p}=0,00000
$$

\section{Critérios de inclusão}

I. Carcinoma de próstata localizado diagnosticado por biópsia através da ultra-sonografia transretal da próstata e tratados por prostatectomia radical retropúbica.

2. Biópsias em sextante, com retirada de pelo menos seis amostras de tecido.

\section{Critérios de exclusão}

Fragmentos de próstata obtidos por outros meios (biópsias perineais, transretais ou por ressecção transuretral da próstata) que não sejam através da ultra-sonografia transretal com biópsia.

\section{Critérios de avaliação}

Apercentagem de fragmentos positivos na biópsia da próstata através da ultra-sonografia transretal foi calculada para cada paciente e distribuída em quatro categorias e correlacionada com o resultado do estudo anátomopatológico do espécime da PRR: tumor confinado ao órgão (doença intraprostática) e não confinado ao órgão (doença extraprostática). Grupo I (GI) - 0\% a 25\% de fragmentos positivos nabiópsia

Grupo 2 (G2) - $26 \%$ a $50 \%$ de fragmentos positivos nabiópsia
Grupo 3 (G3) - 51\% a 75\% de fragmentos positivos na biópsia

Grupo 4 (G4) - 76\% a 100\% de fragmentos positivos na biópsia

Os outros dois parâmetros clínicos, PSA pré-operatório e escala de Gleason da biópsia, foram divididos em grupos e correlacionados com doença intraprostática(DIP) eextraprostática (DEP).

\section{PSA}

Grupo I (GI) - 0 - 4,0 ng/ml

Grupo 2 (G2) - 4,I - 10,0 ng/ml

Grupo 3 (G3) - I0,I - 20,0 ng/ml

Grupo 4 (G4) - > 20,0 ng/ml

\section{Escala de Gleason}

Grupo I (GI) - 2 a 4

Grupo 2 (G2) - 5 a 6

Grupo 3 (G3) - 7 a 10

\section{Análise estatística}

A análise estatística da relação entre a percentagem de doença intraeextraprostática com os parâmetros: I. PSA; 2. Escala de Gleason; 3. Percentagem de fragmentos positivos foi efetuada através do teste do qui-quadrado, aplicado sobre uma tabela de contingência.

Considerando a necessidade de comparar a eficiência dos três parâmetros (PSA pré-operatório, escala de Gleason da 
biópsia e percentagem de biópsia positiva) utilizou-se a técnica de análise discriminante, que permite utilizar variáveis quantitativas para discriminar entre grupos previamente conhecidos - doença intra e extraprostática ${ }^{19}$.

\section{Resultados}

PSA pré-operatório versus doença intra e extraprostática

Na primeira análise, o antígeno prostático específico foi correlacionado com a doença intraprostática e extraprostática, mostrando uma interação altamente significativa $(p=0,00000)$ entre estes fatores (Tabela I). Já que a relação se inverte na quarta categoria (Grupo 4), podemos concluir que esta é responsável pela interação observada.

Houve diferença estatisticamente significativa entre os grupos:

$G 1$ versus $G 2 p=0,0233$

$G 2$ versus $G 3 p=0,0009$

$G$ I versus $G 3 p=0,0002$

$G 2$ versus $G 4 p=0,0000$

$G 1$ versus $G 4 p=0,0000$

$G 3$ versus $G 4 p=0,0573$

Analisando o G4 em relação aos outros grupos, notamos que existe diferença bastante, significativa quando comparado com os grupos $\mathrm{Gl}$ e $\mathrm{G} 2(\mathrm{p}=0,0000)$. No entanto, menos significativa com relação ao $\mathrm{G} 3$ $(p=0,0573)$.

\section{Escala de Gleason da biópsia versus doença intra e extraprostática}

Nesta segunda análise a escala de Gleason foi correlacionada com a presença de doença intra e extraprostática, observando-se, também, uma interação altamente significativa ( $p=0,00000)$ entre estes fatores (Tabela 2). Já que a relação se inverte na terceira categoria (G3), podemos concluir que esta é responsável pela interação observada.

Houve diferençaestatisticamentesignificativa entre os grupos:

$G 1$ versus $G 2 p=0,0124$

$G$ I versus $G 3 p=0,0000$

$G 2$ versus $G 3 p=0,000$ I

Percentagem de fragmentos positivos na biópsia prostática versus doença intra e extraprostática

$\mathrm{Na}$ terceira análise, a percentagem de

\begin{tabular}{lcccccc}
\hline \multicolumn{6}{c}{ Tabela 3 } & Correlação entre a percentagem de fragmentos positivos na biópsia prostática \\
e o câncer intra e extraprostático & & \\
\hline Grupos & Frag.Pos. & DIP & & DEP & n \\
& $\%$ & & & & & \\
& & $n$ & $\%$ & $n$ & $\%$ & \\
G1 & & $n$ & $\%$ & $n$ & $\%$ & \\
G2 & $0-25$ & 95 & 73,08 & 35 & 26,92 & 130 \\
G3 & $26-50$ & 161 & 73,18 & 59 & 26,82 & 220 \\
G4 & $51-75$ & 53 & 61,63 & 33 & 38,37 & 86 \\
Total & $76-100$ & 37 & 43,02 & 49 & 56,98 & 86 \\
\hline
\end{tabular}

\% Frag. Pos.-percentagem de fragmentos positivos na biópsia prostática;DIP-doença intraprostática;DEP-doença extraprostática; n-númerode pacientes; $p$-nível de significância

$$
\text { Pearsonqui-quadrado } \quad p=0,00000
$$

Tabela 4 - Ordem de eficiência dos parâmetros PSA pré-operatório, escala de Gleason e percentagem de biópsias positivas em prever doença intra e extraprostática

$\begin{array}{ccc}\text { Ordem de eficiência } & \text { Variável } & \text { Nível de significância }(\mathbf{p}) \\ 1^{\circ} & \text { PSApré } & 0,000000 \\ 2^{\circ} & \text { escaladeGleason } & 0,000003 \\ 3^{\circ} & \text { \%Frag.Pos. } & 0,000574\end{array}$

PSApré-PSA pré-operatório

escala de Gleason-escala de Gleason dabiópsia

\% Frag. Pos. - percentagem defragmentos positivos na biópsia prostática

Gráfico I - Função discriminante aplicada sobre o PSA, a escala de Gleason da biópsia e a percentagem de biópsias positivas em relação à doença intraprostática (intra) e extraprostática (extra)

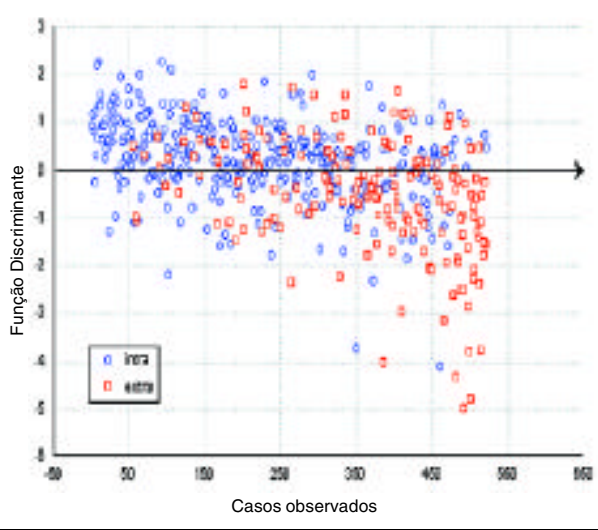

fragmentos positivos da biópsia prostática versus doença intra e extraprostática também mostrou uma interação altamente significativa $(p=0,00000)$ entre estes fatores (Tabela 3). Já que a relação se inverte na quarta categoria (G4), podemos concluir que esta é responsável pela interação observada.
Com relação à diferença estatisticamente significativa entre os grupos notamos:

$G 1$ versus $G 2 p=$ iguais

$G 2$ versus $G 3 p=0,0301$

$G$ I versus $G 3 p=0,0448$

$G 2$ versus $G 4 p=0,0000$

$G$ I versus $G 4 p=0,0000$

$G 3$ versus $G 4 p=0,0068$ 
Estudo comparativo entre os parâmetros PSA pré-operatório, escala de Gleason da biópsia e percentagem de biópsias positivas para estabelecer a ordem de eficiência de cada variável em prever doença intra e extraprostática

A análise discriminante mostrou a ordem de entrada das variáveis no modelo, o que expressa a importância relativa de cada uma na discriminação dos grupos intra e extraprostático. Aordem decrescente de eficiência das variáveis utilizadas como discriminanteséa seguinte: PSA pré-operatório, escala de Gleason e percentagem de biópsias positivas. Todas as variáveis mostraram significância $(\mathrm{p}<0,000)$ ao discriminar os grupos (Tabela 4).

\section{Discussão}

O nosso trabalho tem a característica de valorizar os achados da biópsia prostática guiadas por ultra-sonografia transretal em pacientes com câncer de próstata tratados por prostatectomia radical retropúbica. Trata-se de estudo, ainda não descrito na literatura, que abrange um número expressivo de pacientes ( 522 casos) e a utilização da percentagem de fragmentos positivos da biópsia prostática como parâmetro único. Como resultado principal, demonstrou que mais de $75 \%$ de fragmentos positivos na biópsia prostática estariam associados a fatores de mau prognóstico, especificamente presença de doença extraprostática.

$O$ estudo da percentagem de fragmentos positivos na biópsia prostática permitiu que demonstrássemos uma preponderância de doença intraprostática sobre a extraprostática nos grupos I, 2 e 3 . A relação se inverteu quando a biópsia prostática revelou mais de $75 \%$ de fragmentos comprometidos por tumor (Grupo 4), notando-se doença extraprostática em $57 \%$ dos casos ( $G 3$ versus G4 $p=0,0063$ ).

Alguns resultados semelhantes aos nossos foram observados por outros autores, que utilizaram a percentagem de biópsias positivas como um dos parâmetros de estudo para melhorar o estagiamento clínico $11,20,21$. Algumas diferenças numéricas baseiam-se, possivelmente, no tamanho da nossa amostra. Praticamente todos os autores citados trabalharam com múltiplos parâmetros e, em média, com 100 pacientes portadores de carcinoma clinicamente localizado, ao passo que o nosso grupo inclui 522 pacientes com doença localizada.

Além da influência da percentagem de fragmentos de biópsia positivos, demonstramos em nosso estudo o valor da correlação entre o PSA pré-operatório (Tabela I) e a escala de Gleason da biópsia (Tabela 2) com a doença intra e extraprostática. Estes achados, naverdade, não são originais e têm sido amplamente divulgados na literatura ${ }^{10,11,12,21,22,23,24}$.

Devido ao fato da biópsia prostática nem sempre refletir com exatidão as características da neoplasia, ainda existem controvérsias na literatura quanto à utilização dos achados da biópsia como parâmetro ideal na diferenciação de doença intra ou extraprostática.

A literatura carece de trabalhos que utilizaram um grande número de pacientes para avaliar o valor da percentagem de fragmentos positivos como parâmetro único para detectar doença extraprostática.

A utilização das análises feitas anteriormente não permite decidir qual dos três parâmetros (PSA, escala de Gleason da biópsia e percentagem de biópsias positivas) seria 0 melhor, uma vez que estes foram utilizados independentemente uns dos outros e sobre dados categóricos (as medidas de PSA, por exemplo, foram categorizadas em quatro faixas, rebaixando-se desse modo a escala de medição).

Considerando a necessidade de comparar a eficiência destes três parâmetros, utilizou-se a técnica de análise discriminante que permite utilizar variáveis quantitativas para discriminar entre grupos previamente conhecidos (doença intra e extraprostática). Tomou-se, então, as medidas de PSA, escala de Gleason da biópsia e percentagem de biópsias positivas (caso a caso, e não mais em grupos) e executou-se uma análise discriminante que permitisse avaliar como estes três parâmetros teriam influência na separação dos grupos conhecidos de pacientes classificados como tendo doença intra e extraprostática (Gráfico I).

Aordem de entrada das variáveis no modelo, que expressa a importância relativa de cada variável na discriminação dos grupos fornecidos, foi: $\left.1^{\circ}\right)$ PSA, $2^{\circ}$ ) escala de Gleason da biópsia e $3^{\circ}$ ) percentagem de biópsias positivas (Tabela4). Todas as variáveis mostraram significância ao discriminar os grupos $(p<0,000)$.
No Gráfico I notamos um grupamento de casos em torno da seta, com uma densidade maior de doença intraprostática (intra) acimae extraprostática (extra) abaixo desta. Logo, denuncia que há outros fatores envolvidos na discriminação dos grupos (doença intra e extraprostática) que ainda não foram incluídos no modelo.

As implicações do nosso trabalho podem servistas de diferentes maneiras. Em primeiro lugar, o nosso estudo permitiu definir números nacionais para apoiar o estudo de pacientes com câncer localizado da próstata e futuras publicações. Estes resultados merecem credibilidade tendo em vista a rígida seleção e o grande número de pacientes incluídos.

Ademais, demonstramos que a percentagem de fragmentos positivos na biópsia prostática permite predizer com maior segurança a faixa de risco de doença extraprostática. Isto significa que, nos pacientes com mais de $75 \%$ dos fragmentos de biópsia positivos, devem ser procuradas formas inovadoras de tratamento, já que a cirurgia radical tem pouca chance de ser eficiente.

Como proposta futura e partindo dos achados do presente estudo, fica claro que outros fatores prognósticos devem ser explorados em câncer localizado da próstata. Percentagem elevada de fragmentos de biópsia positivos, PSA pré-operotório e valores da escala de Gleason permitem caracterizar a agressividade desses tumores, mas não são absolutos, exigindo critérios adicionais de prognóstico para podermos estabelecer com precisão as características biológicas das neoplasias da próstata.

\section{SUMMARY}

EXTRAPROSTATIC DISEASE PREDICTION IN PATIENTS WITH CARCINOMA OF THE PROSTATE: ROLE OF PSA, PROSTATIC BIOPSY FRAGMENTS PERCENTAGE AND GLEASON SCORE

OBJECTIVE. To valuate the behavior of the prostatic biopsy fragments percentage, guided by transrectal ultrasonography in extraprostatic disease prediction in patients with localized adenocarcinoma of the prostate gland and, also, to compare the efficiency of this pattern with them got by the valuation of PSA and preoperative Gleason score.

Methods. This retrospective noncontrolled study consisted of 522 patients 
with adenocarcinoma located in the prostate. They were submitted to surgical treatment through retropubic radical prostatectomy. The ages of the patients ranged from 42 to 76 years with a mean of 62.44 years. All of them were submitted to transrectal ultrasonography with prostatic biopsy (direct of the lesion and/or suspected area and sextant) previous to the radical surgical treatment. The 522 patients were divided in groups according to the positive fragments found by the biopsy, what was correlated with the anatomicpathologic findings of intraprostatic disease (limited to the gland) and extraprostatic (invasion periprostatic adiposity and/or the bladder neck and/or the seminal vesicles and/or positive pelvic lymph nodes) of the surgical specimen.

RESULTS. Regarding the analysis of the positive fragments percentage, the groups GI (0-25\%), G2 (20-50\%) and G3 (5I$75 \%)$ showed an incidence of the intraprostatic disease two and three times greater than the extraprostatic one. However, when more than $75 \%$ of the biopsy fragments were positive (G4, 76-100\%), the relation inverted occurring a predominance of the extraprostatic disease over the intraprostatic. In this group $56.98 \%$ of the patients showed extraprostatic disease. There was a statistically significant difference of the extraprostatic disease between the groups G3 and G4 (p 0.0068).

CONCLUSION. When we compared the three inconstant to evaluate the efficiency order anticipating extraprostatic disease, we verified that the preoperative PSA was the most discriminated $(p=0.000000)$ followed by the Gleason score of prostatic biopsy $(p=$ 0.000003 ) and by the positive biopsies percentage $(p=0.000574)$. [Rev Assoc Med Bras 2003; 49(3): 250-4]

KeY words. Prostate. Prostate-specific antigen. Prostatic neoplasm. Biopsy.

\section{RefERÊNCIAS}

I. Brawer MK, Chetner MP, Beatie J, Buchner DM, Vessella RL, Lange PH. Screening for prostatic carcinoma with prostate specific antigen. J Urol 1992; I47:84I-5.

2. Catalona WJ, Smith DS, Ratliff TL, Basler JW. Detection of organ-confined prostate cancer is increased through prostate specific antigen based screening. JAMA 1993; 270:948-54.

3. Carter HB, Coffey DS. The prostate: an increasing medical problem. Prostate 1990; 16:39-48.

4. Wingo PA, Tong T, Bolden S. Cancer statistic. Cancer J Clin 1995; 45:8-31.

5. Partin AW, Oesterling JE. The clinical usefulness of prostate specific antigen: update 1994. J Urol 1994; 152: 1358-68.

6. Stamey TA, Kabalin JN. Prostate specific antigen in the diagnosis and treatment of adenocarcinoma of the prostate. I. Untreated. JUrol 1989; |41:1070-5.

7. Hering FLO, Srougi M. Adenocarcinoma de próstata: correlação entre o valor sérico do PSA pré-operatório, doença extraprostática e progressão do tumor. J Bras Urol 1995; 21:5I-3.

8. Partin AW, Yoo J, Carter HB, Pearson JD, Chan DW, Epstein Jl et al. The use of prostate specific antigen, clinical stage a Gleason score in men with localized prostate cancer. J Urol 1993; 150:110-4.

9. Danziger M, Shevchuk M, Antonescu C, Matthews GJ, Fracchia JA. Predictive accuracy of transrectal ultrasound-guided prostate biopsy: correlations to matched prostatectomy specimens. Urology 1997; 49:863-7.

10. Narayan P, Gajendran V, Taylor SP, Tewari A, Presti JC, Leidich $R$ et al. The role transrectal ultrasound-guided biopsy based staging, preoperative serum prostate specific antigen, and biopsy Gleason score in prediction of final pathologic diagnosis in prostate cancer. Urology 1995; 46:205-12.

II. Sebo TJ, Bock BJ, Cheville JC, Lohse C, Wollan $P$, Zincke $H$. The percent of cores positive for cancer in prostate needle biopsy specimens is strongly predictive of tumor stage and volume at radical prostatectomy. J Urol 2000; 163:174-8.

12. Wills ML, Sauvageot J, Partin AW, Garganus R, Epstein JI. Ability of sextant biopsies to predict radical prostatectomy stage. Urology 1998; 51:759-64.

13. Ravery V, Schmid HP, Toublanc M, BocconGibod L. Is the percentage of cancer in biopsy cores predictive of extracapsular disease in $\mathrm{TI}$ - T2 prostate carcinoma? Cancer 1996; 78:1079-84.

14. Terris MK, McNeaLJE, Stamey TA. Detection of clinically significant prostate cancer by transrectal ultrasound-guided systemic biopsies. J Urol 1992; 148:829-32.

15. Terris MK. Sensitivity and specificity of sextant biopsies in the detection of prostate cancer: preliminary report. Urology 1999; 54:486-9.

16. Manseck A, Froehner M, Oehlschlaeger S, Hakenberg $\mathrm{O}$, Friedrich $\mathrm{K}$, Theissig $\mathrm{F}$ et al. Is systematic sextant biopsy suitable for the detection of clinically significant prostate cancer? Urol Int 2000; 65:80-3.

17. Srougi M, Simon SD. Câncer da próstata. In: Srougi M, editor. Câncer urológico. São Paulo: Editora Platina; 1 996. p.28I-359.

18. International Union Against Cancer. In: Sobin $\mathrm{LH}$, Wittekind $\mathrm{CH}$, editors, TNM classification of malignant tumors. $5^{\text {th }}$ ed. New York: Jonh Wiley \& Sons; 1997. p. I70-3.

19. Hair J, Anderson RE, Tatham RL. Multivariate data with readings. New York: Macmillan, 1995.

20. Peller PA, Young DC, Marmaduke DP, Marsh WL, Badalament RA. Sextant prostate biopsies. Cancer 1995; 75:530-8.

21 . Borirakchanyavat S, Bhargava V, Shinohara K, Toke A, Carrol PR, Presti JC. Systematic sextant biopsies in the prediction of extracapsular extension at radical prostatectomy. Urology 1997; 50:373-8.

22. Egawa S, Suyama K, Matsumoto K, Satoh T, Uchida T, Kuwao S et al. Improved predictability of extracapsular extension and seminal vesicle involvement based on clinical and biopsy findings in prostate cancer in japanese men. Urology 1998; 52:433-40.

23. Tigrani VS, Bhargava V, Shinohara K, PrestiJC. Number of positive systematic sextant biopsies predicts surgical margin status at radical prostatectomy. Urology 1999; 54:689-93.

24. Gohji K, Okamoto M, Takenaka A, Fujii A. Predicting the extent of prostate cancer using combination of systematic biopsy and serum prostate-specific antigen in japanese men. BJU Int 1999; 83:39-42.

Artigo recebido: 23/05/03 Aceito para publicação: 29/05/03 